\title{
Resistance Estimation for Temperature Determination in PMSMs through Signal Injection
}

\author{
Simon D. Wilson, Geraint W. Jewell and Paul G. Stewart \\ Electrical Machines and Drives Research Group \\ Department of Electronic and Electrical Engineering \\ The University of Sheffield \\ Mappin Street, S1 3JD
}

\begin{abstract}
Real-time thermal management of electrical machines relies on sufficiently accurate indicators of temperature within a machine. One indicator of temperature in a permanentmagnet synchronous motor (PMSM) is the stator winding resistance. Detection of PMSM winding resistance in the literature has been made on machines with relatively high resistances, where the resistive voltage vector is significant under load. This paper describes a technique applied to sense the winding resistance where the resistance is low and hence the resistive is voltage difficult to detect. A current injection method is applied which enables the resistance to be determined, and hence the winding temperature in non-salient machines. This method can be applied under load, and in a manner that does not disturb shaft torque, or speed. The method is able to distinguish between changes in the electro-motive force (EMF) constant and the resistive voltage. Simulated results on an experimentally verified model illustrate the effectiveness of this approach.
\end{abstract}

\section{INTRODUCTION}

Brushless permanent-magnet synchronous machines are the preferred technology in many applications due to their high specific power ratings, efficiencies and speed range. In common with other motors, their power output is often limited by the maximum operating temperature of the machine. For intermittent duty cycle operation, a steady-state method of sizing a machine to achieve a given rating becomes potentially very conservative. A method that fully exploits the thermal capacity of the machine is able give a transient power capability that is several times the continuous rating, especially if the ambient temperature is variable. Temperature monitoring can be achieved using embedded thermal sensors, but these increase the wiring complexity [1].

Considerable research has been published on the estimation of stator resistance in induction machines for the improvement of sensorless speed and torque control [2]-[8] and thermal monitoring [9]. On-line stator resistance estimation has also been applied to PMSMs to improve sensorless control [10], but these estimates are not sufficiently accurate for thermal monitoring.

Sensorless thermal monitoring has been demonstrated [11], protecting a 200W PMSM from coolant failure. The optimal speed range for temperature estimation was found to be 1000 - $2000 \mathrm{rpm}$., above which the induced electromotive force (EMF) dominates over the resistive volts drop. In this method no mechanism exists to separate changes in the EMF constant from a change in the resistive voltage detected.

DC injection can be used to directly obtain the resistance from the voltage and current through a winding. Either injection of a DC bias into the stator supply voltage can be used [12], or into the neutral of a wye-connected machine with a capacitor bank [13]. Injection of a DC bias causes pulsating torque output, and both methods dissipate power in the machine.

The techniques presented in this paper are applied to the problem of temperature estimation for thermal management of PMSMs with low resistance, and under closed loop control. The injected current method is used to temporarily boost the resistive voltage available for detection. The well-known $d q$ transform [14] is used to study control techniques of PMSMs, and hence the study of current injection here is also in the $d q$ reference frame.

\section{PMSM DQ AXIS MODEL}

The $d q$ circuit model of a PMSM can be modelled

$$
\begin{aligned}
{\left[\begin{array}{l}
v_{d} \\
v_{q}
\end{array}\right]=} & {\left[\begin{array}{cc}
R_{d} & -\omega L_{q} \\
\omega L_{d} & R_{q}
\end{array}\right]\left[\begin{array}{c}
i_{d} \\
i_{q}
\end{array}\right] } \\
& +\left[\begin{array}{cc}
L_{d} & 0 \\
0 & L_{q}
\end{array}\right] \frac{d}{d t}\left[\begin{array}{l}
i_{d} \\
i_{q}
\end{array}\right]+\left[\begin{array}{c}
0 \\
K_{e} \omega_{m}
\end{array}\right]
\end{aligned}
$$

where $R_{d}, R_{q}, L_{d}, L_{q}$ are the $d$ and $q$ axis resistances and inductances respectively, $K_{e}$ is the back-emf constant, $\omega$ is the electrical frequency and $\omega_{m}$ is the rotor velocity.

\section{INJECTION CURRENT}

In principle, the sensing current can be injected into any combination of phases in a variety of relative proportions. By way of example, Fig 1(a) shows a case in which the current is injected into phase 'A' returning entirely through phase ' $\mathrm{B}$ ', while in Fig. 1(b) the current is again injected into phase ' $A$ ' but returns in equal proportions through phases ' $\mathrm{B}$ ' and ' $\mathrm{C}$ '. Both these paths can be represented by a current vector, Fig. 2 with $d q$ components

$$
\begin{aligned}
& i_{d s}=F \cos \left(\theta_{F}\right) \\
& i_{q s}=F \sin \left(\theta_{F}\right)
\end{aligned}
$$




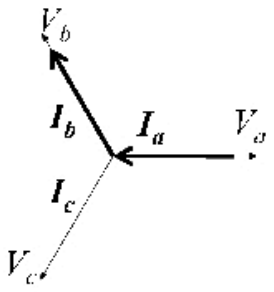

(a)

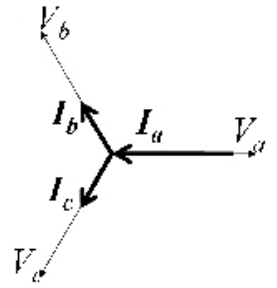

(b)
Fig. 1. Current paths

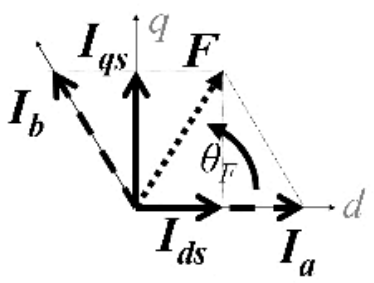

Fig. 2. General current vector

The injected current causes a magneto-motive force to be present in the airgap of the machine. An additional rotating current controlled vector will be present when the motor comprises part of a drive system and is producing torque. When referred to the rotor stationary frame $(q d r)$, the injection current vector is represented as rotating

$$
\begin{aligned}
& i_{d r}=F \cos \left(\theta_{F}-\theta\right) \\
& i_{q r}=F \sin \left(\theta_{F}-\theta\right)
\end{aligned}
$$

Further we obtain the following equations of $d$ and $q$ axis voltage by substitution of $(4,5)$ in (1)

$$
\begin{aligned}
v_{d r} & =R_{d} I_{d r}+2 \omega L i_{q r} \\
v_{q r}-K_{e} \omega_{m} & =R_{q} I_{q r}-2 \omega L i_{d r}=v_{q r}^{\prime}
\end{aligned}
$$

$v_{q r}^{\prime}$ is decoupled from the back-emf voltage by subtracting $K_{e} \omega_{m}$. This parameter dependence is undesirable, and can be avoided by the measurement procedure as described in Section VIII. For a non-salient machine $L_{d}=L_{q}=L$, and assuming the winding resistances are equal $R_{d}=R_{q}=R$, the voltage vector diagram of Fig. 3 is formed in the stator stationary $(q d s)$ frame.

The injection scheme can thus measure the resistance and inductance of the PMSM. The $d$ and $q$ axis voltages can be resolved onto injection axes $v_{q i}, v_{d i}$ and the quantities $R F$ and $2 \sqrt{2} \omega L F$ separated

$$
\begin{aligned}
v_{d i} & =2 \sqrt{2} \omega L F=v_{d s} \sin \theta_{F}-v_{q s}^{\prime} \cos \theta_{F} \\
v_{q i} & =R F \quad=v_{d s} \cos \theta_{F}+v_{q s}^{\prime} \sin \theta_{F}
\end{aligned}
$$

The injection currents and detected voltages comprise the DC injection scheme, the injection voltage in the $q d s$ reference frame is stationary.

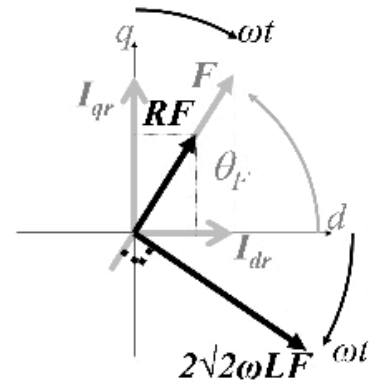

Fig. 3. Injection voltages in $q d r$ frame.

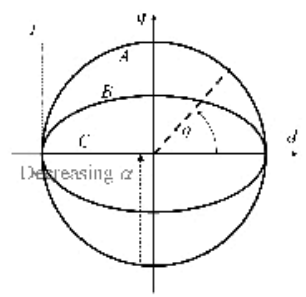

Fig. 4. Injection current loci

\section{TORQUE RIPPLE REDUCTION}

DC injection contributes to the $d q$ axis currents, of which the $q$-axis component is torque producing:

$$
T_{e m}=K_{t} i_{q}
$$

where $T_{e m}$ is the torque and $K_{t}$ is the torque-constant of the PMSM

Reduction of the $q$ component of the injection current causes its torque contribution to decrease. Machine speed deviation can then be reduced. The current loci for DC injection ' $A$ ', reduced torque injection ' $B$ ' injection and zero-torque injection ' $C$ ' are shown Fig. 4. Reduction of the $q$ axis current by the regulation factor ' $\alpha$ ' causes a corresponding reduction in torque (10).

The following equations describe the reduced-torque injection currents and torque production:

$$
\begin{aligned}
i_{d r} & =F \cos \left(\theta_{F}-\theta\right) \\
i_{q r} & =\alpha F \sin \left(\theta_{F}-\theta\right) \\
\tilde{T}_{e m} & =\alpha F \cos \left(\theta_{F}-\theta\right)
\end{aligned}
$$

where $\tilde{T}_{e m}$ denotes the pulsating torque produced by injection.

When $\alpha=0$, no torque is produced. This is a desirable characteristic, hence further investigation is into this case of signal injection is merited.

\section{Optimum Estimation}

To give optimum temperature estimation per amp of injected current, the greatest and clearest resistive signal voltage must be produced. Analysis of the injection currents and induced 


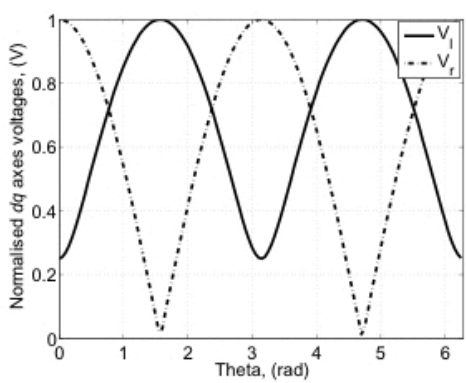

Fig. 5. Normalised magnitudes of injection voltages for $\theta_{f}=90^{\circ}$

voltages produces the following voltage equations for $\alpha=0$

$$
\begin{aligned}
& {\left[\begin{array}{c}
v_{d s} \\
v_{q s}
\end{array}\right]=} \\
& {\left[\begin{array}{c}
F R \cos \left(-\theta+\theta_{f}\right) \cos (\theta)-\omega L F \\
\left(-4 \sin \left(-\theta+\theta_{f}\right) \cos (\theta)+\cos \left(-\theta+\theta_{f}\right) \sin (\theta)\right) \\
F_{s} R \cos \left(-\theta+\theta_{f}\right) \sin (\theta)+\omega L F \\
\left(\cos \left(-\theta+\theta_{f}\right) \cos (\theta)+4 \sin \left(-\theta+\theta_{f}\right) \sin (\theta)\right)
\end{array}\right]}
\end{aligned}
$$

Separating the resistive and inductive components of (14) and analysing the magnitude and phase of each, the resistive voltage is found to be maximum at $-\theta+\theta_{f}=0$. The corresponding inductive voltage is a minimum at this point, with the two voltages being orthogonal. As rotor position moves away from $-\theta+\theta_{f}=0$, the angle between the two voltages becomes non-orthogonal making it more likely that errors in rotor position measurement will adversely affect estimation, commuting some of the greater inductive voltage onto the resistive voltage.

Therefore the optimum estimation for resistance takes place at $-\theta+\theta_{f}=0$. Both resistance and inductance can be found at this point. The inductive signal is a maximum at $-\theta+$ $\theta_{f}+90^{\circ}=0$, and where the inductance may be the quantity of interest the greatest signal voltage is found at this point Fig. 5.

At rated speed, the time period during which estimation can be performed is small. To increase the possible arc of current injection the estimator system must separate the two quantities when not orthogonal

$$
\left[\begin{array}{l}
V_{r} \\
V_{l}
\end{array}\right]=\left[\begin{array}{c}
-\frac{V \sin \left(\theta_{l}-\theta_{v}\right)}{\sin \left(-\theta_{l}+\theta_{r}\right)} \\
\frac{V \sin \left(-\theta_{v}+\theta_{r}\right)}{\sin \left(-\theta_{l}+\theta_{r}\right)}
\end{array}\right]
$$

where $V, \theta_{v}$ are the magnitude and angle of the sum of the resistive and inductive voltages in $q d s$ frame, and $\theta_{r}, \theta_{l}$ are the angles of the resistive and inductive voltages respectively. $\theta_{r}, \theta_{l}$ can be calculated (14) and depend on rotor position but not the magnitudes of $R, L$. The denominator is non-zero during injection.

\section{INJECTION CURRENT PROFILE}

It is clear that since estimation is most accurate at $-\theta+$ $\theta_{f}=0$, there is no benefit to injecting current for a long

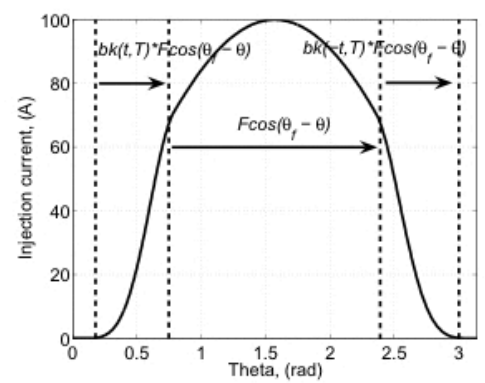

Fig. 6. Injection current profile for $\theta_{f}=90^{\circ}$

arc length of position $\theta$. Rather it should be concentrated in the region of optimum estimation. The required current (11) is a portion of a sinusoid. To avoid exciting the system with electrical oscillations that may be caused using a rectangular or trapezoidal window, the injection current is modified by the 'Blackman' window function [15] at turn-on and turn-off

$$
\begin{aligned}
b k(t, T) & =0.42+0.5 \cos \left(\frac{\pi t}{T}\right)+0.08 \cos \left(2 \frac{\pi t}{T}\right) \\
\max \left(\frac{d i}{d t}\right) & =\frac{1.81 F}{T} \\
\int_{-T}^{T} i d t & =0.84 F T
\end{aligned}
$$

where $b k$ is the window function, $t$ is elapsed time, and $T$ is the length of the window.

Multiplying the window function in a piecewise manner with the injection current (11) realises the injection current profile Fig 6 . The voltage required to drive the current profile can be calculated using the maximum rate of change of current (17).

\section{POWER DisSipation AND INVERTER RATING}

The $d$-axis injection current flows through equivalent $d$-axis resistance $R_{d}$ causing power dissipation

$$
P_{a v}=\frac{R_{d}}{T_{m}}\left(\frac{2 F}{\omega} \sin \left(\frac{\omega t_{i}}{2}\right)+0.84 F T\right)^{2}
$$

where $t_{i}$ is the length of the time window of sinusoidal injection, and $T_{m}$ is the period between successive injections.

For $T=t_{i}=0.001 \mathrm{~s}, T_{m}=60 \mathrm{~s}$ and the parameters of Table I, $P_{a v}=0.6 \mathrm{~mW}$. For most motors this represents a negligible power loss when compared to motor rated output power. The inverter volt-amp rating will need to be increased if estimation is desired at motor rated speed. The extra voltage and current rating required during injection can be calculated $(20,21)$.

$$
\begin{aligned}
V_{i} & =F \sqrt{\left(\frac{1.81 L_{d}}{T}\right)^{2}+R^{2}} \\
I_{i} & =\frac{2}{3} F
\end{aligned}
$$

Given a maximum voltage available for injection, and a particular machine, the maximum achievable injection current is calculated, Fig. 7. 


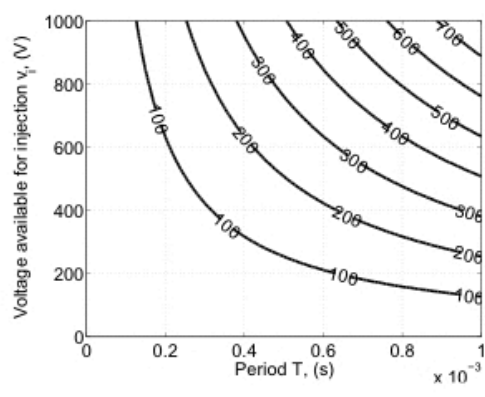

Fig. 7. Maximum current available for injection with $L_{d}=0.7 \mathrm{mH}$ and $R=10 \mathrm{~m} \Omega$

\section{SEPARATION OF INJECTION VOLTAGE FROM NORMAL MOTOR OPERATING VOLTAGE}

To apply the scheme when the motor is operating under closed loop control the normal motor operating voltages must be separated from the injection voltages. Vector motor controllers operate on the principal of referring quantities to the rotor frame. The magnitude and phase of motor current is adjusted to maintain maximum torque per amp and the correct amount of flux-weakening (FW) in the constant power region of the motor's torque-speed characteristic. The rotor referred axes currents are integral to operation and at constant speed and torque output the referred currents become mean levels

$$
\begin{aligned}
I_{q} & =\frac{1}{k_{T}}\left(J \frac{d \omega_{m}}{d t}+B \omega_{m}+T_{L}\right) \\
I_{d} & =0, \text { in non-FW region }
\end{aligned}
$$

When in FW region the motor axes currents are defined by the FW control scheme, but will still consist of mean levels at constant torque and speed. Many practical drive systems cannot achieve both criteria at once, however the behaviour of the referred axes voltages can be linearised for small time intervals by latching the motor torque command to some value during the current injection procedure. Motor speed may still vary, but in a linear manner if the load torque remains constant during the small injection time period.

\section{A. Sinusoidal back-EMF}

If the machine back-EMF is a pure sinusoid consisting of a fundamental component only then the rotor referred EMF is a mean voltage without harmonic content. To separate the injection quantities requires detection of the mean level, which can be achieved by a linearising method. The linearisation consists of sampling the axes voltages pre and post-injection during the time window for which the torque command is latched. The pre and post samples are aggregated to a single representative point and interpolation performed between them to give the mean axes voltages without the injection signal, Fig. 8 .

The linearisation method is thus able to provide a signal for estimation during motor acceleration or deceleration, the cost being a small period during which the motor is unable to respond to speed or torque command changes. It also has

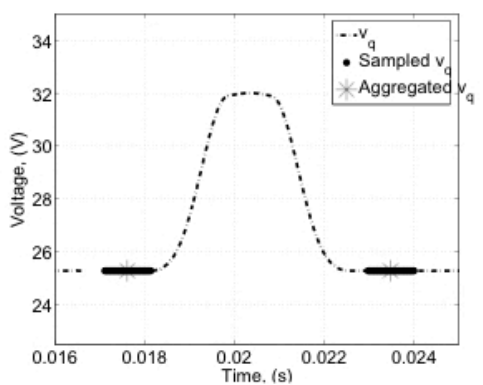

Fig. 8. Linearisation of axis voltage at $\omega=100 \mathrm{rads}^{-1}$

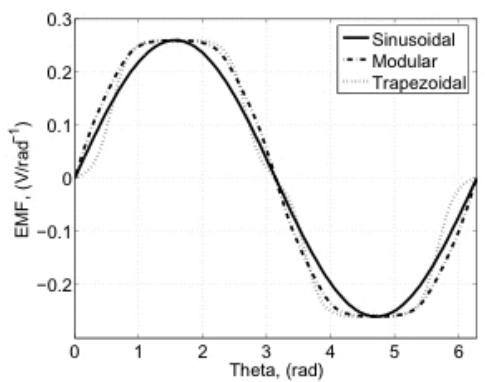

Fig. 9. Back-EMF profiles

good noise rejection, as the number of samples taken for each aggregated point can be large.

\section{B. Non-sinusoidal back-EMF}

Although high-precision AC servomotors are designed to have an EMF waveform which is a close approximation to a sinusoid to give smooth and high bandwidth torque control. Due to machine design constraints this ideal waveform cannot be achieved and the EMF waveform can contain significant harmonics. When considered in the rotor frame the harmonics are lowered by one harmonic number. Fig. 9 shows an ideal sinusoid and two EMF waveforms of the same peak voltage which are typical of those observed in many practical machines. The 'Sinusoidal', 'Modular' and 'Trapezoidal' machines contain $100 \%, 89 \%$ and $77 \%$ fundamental components respectively.

The referred harmonics interfere with the injection voltage measurement and in the case of the trapezoidal machine introduce large amplitude oscillations when compared to the injection signal voltages. The linearisation method of distilling the injection signal from the axes voltages therefore becomes inaccurate. Detection of the mean level of axis voltage cannot provide a sufficiently precise method of separating the normal operation since the mean induced harmonic voltage is nonzero over the period of the injection, Fig. 10. Increasing the injection period to match that of the lowest harmonic is an undesirable solution since at low speeds the motor torque control must be disabled for an extended period of time.

Since the induced harmonic voltage amplitude is small in comparison to the motor rated voltage an estimation procedure 


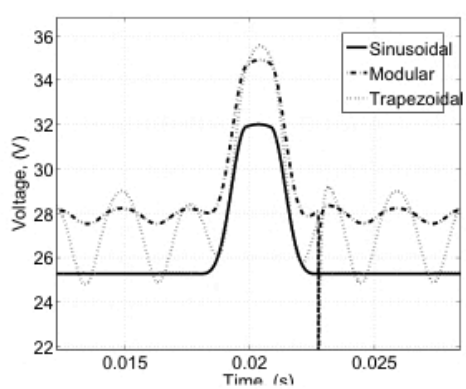

Fig. 10. D-axis voltage for EMF profiles at $\omega=100 \mathrm{rads}^{-1}$ Note: Transient spike generated as torque-hold window ceases.

for calculating the harmonic voltage for a particular angle will have significant errors due to finite transducer accuracy, quantisation and system noise. Therefore any estimate of the oscillating voltage may not significantly improve the separation of the injection signal from the motor voltage.

A filter method applied in the $d q$ frame could eliminate the greatest amplitude harmonics, however the harmonic frequencies change continuously as the motor speed changes and hence the filter would need to be variable. There is also the problem of filter time delay affecting the measurements since the measurement is preceded by a large voltage which drives the injection current profile as (20).

The application of successive phase shifts to cancel each harmonic by applying $180^{\circ}$ phase shifts to each is also an unsatisfactory solution since to avoid interference the injection signal must not occupy a greater angle than the period of the highest harmonic to be canceled. If the highest harmonic to be canceled is the $12 \mathrm{th}$, then only a $15^{\circ}$ arc is available for injection. With a $20 \mathrm{kHz}$ PWM converter and an electrical frequency of $200 \mathrm{~Hz}$ only 4 voltage measurements can be made, not enough for an accurate voltage measurement given the noisy data problem. Therefore to reasonably limit the errors introduced to the voltage measurement the linearising method can be applied only to machines with an EMF which is a good approximation to a pure sine wave.

\section{Simulation}

Results have been obtained using a 'Simulink' model of a PMSM drive system and controller. The motor control system consists of PID speed control loop, Model-Reference Vector and Feedback Linearising controllers [14]. The linearising controller matches axis current to the motor demand current, a vector controller calculates required $I_{d}^{*} \& I_{q}^{*}$ to satisfy the demanded torque output at the current motor velocity. The injection current demands are summed with the vector control current demands and sent to the current controller. The system parameters are defined as Table I. The resistive voltage under load is small, $0.41 \mathrm{~V}$ for the torque setting and winding resistance. This represents a fraction of the rated voltage $(0.17 \%)$.

Noise has been added to the injection signals prior to estimation to represent the effect of quantisation of the voltage
TABLE I

SySTEM PARAMETERS

\begin{tabular}{|l|l|l|}
\hline$\omega_{\text {set }}$ & $100 \mathrm{rads}^{-1}$ & Speed set-point \\
\hline$T_{\text {set }}$ & $19.6 \mathrm{Nm}^{-1}$ & Torque set-point \\
\hline$K_{t}$ & 0.48 & Torque constant \\
\hline$K_{e}$ & 0.26 & EMF constant \\
\hline$P_{p}$ & 4 & Pole-pairs \\
\hline$V_{\text {rated }}$ & $240 \mathrm{~V}$ & Motor rated phase voltage \\
\hline$J$ & $0.0114 \mathrm{~kg} / \mathrm{ms}^{-2}$ & System inertia \\
\hline$R_{d q}$ & $10 \mathrm{~m} \Omega$ & Motor $d q$ resistance \\
\hline$L_{d q}$ & $0.708 \mathrm{mH}$ & Motor $d q$ inductance \\
\hline$F$ & $100 \mathrm{~A}$ & Injection current magnitude \\
\hline$\sigma_{n}$ & $2 \times 10^{-4} V^{2}$ & Covariance of system noise \\
\hline$t_{\text {sim }}$ & $1 \times 10^{-5} \mathrm{~s}$ & Simulation time-step \\
\hline$\theta_{\text {inj }}$ & $45^{\circ}$ & Injection current arc \\
\hline$\theta_{t h}$ & $60^{\circ}$ & Torque-hold arc \\
\hline
\end{tabular}

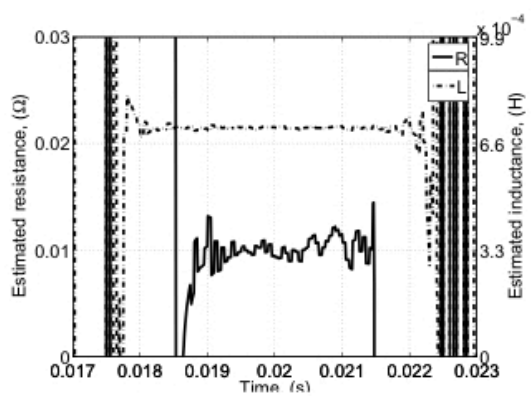

Fig. 11. Estimation signals prior to recursive mean application at $\omega=$ $100 \mathrm{rads}^{-1}$

measurement, and system noise. The recursive mean algorithm [16] has been applied to the estimation signals.

\section{Results}

For the above system the results in Table II were obtained at constant speed and torque output. The difference between the three estimates is a result of the statistical nature of the added noise. Table III estimates have been obtained during acceleration. In Table IV the estimation of resistance is shown to be sensitive to the system noise level. The noise covariances of $\sigma_{n}=2 \times 10^{-5}, \sigma_{n}=2 \times 10^{-1}$ were seen to correspond to pk-pk noise voltages of approximately $0.02 \mathrm{~V}$ and $2 \mathrm{~V}$ respectively.

The estimate of inductance is obtained to a high precision in all results, whilst the resistance estimate shows sensitivity to speed, acceleration and noise. Sensitivity to noise arises as a consequence of the detection of mean levels in the measurements, and can therefore be improved by increasing the length of the data available for estimation. This is achievable by increasing $\theta_{i n j}$ or by decreasing $\omega$. Resistance estimation accuracy degrades at high speed due to the dominance of the inductive voltage in the injection signal (14). In Table II the spread of resistance estimates corresponds to an uncertainty of $2.7^{\circ} \mathrm{C}$ and $5.4^{\circ} \mathrm{C}$ for the sinusoidal and modular machines respectively.

In the case of modular EMF, waveform shown previously in Fig. 9, the estimates are less accurate than the sinusoidal 
TABLE II

RESULTS AT CONSTANT SPEED

\begin{tabular}{|l|l|l|l|}
\hline \multicolumn{4}{|l|}{ Sinusoidal EMF } \\
\hline$\omega_{\text {set }}=100 \mathrm{rads}^{-1}$ \\
\hline Estimate & 1 & 2 & 3 \\
\hline$\hat{R}$ & $0.0104 \Omega$ & $0.0102 \Omega$ & $0.0101 \Omega$ \\
\hline$\hat{L}$ & $0.709 \mathrm{mH}$ & $0.709 \mathrm{mH}$ & $0.709 \mathrm{mH}$ \\
\hline$\omega_{\text {set }}=300$ rads $^{-1}$ \\
\hline$\hat{R}$ & $0.0095 \Omega$ & $0.0130 \Omega$ & $0.0115 \Omega$ \\
\hline Modular EMF & \multicolumn{5}{l}{} \\
\hline$\omega_{\text {set }}=100 \mathrm{rads}^{-1}$ & 2 & 3 \\
\hline Estimate & 1 & 2 & $0.0106 \Omega$ \\
\hline$\hat{R}$ & $0.0112 \Omega$ & $0.0110 \Omega$ & $0.725 \mathrm{mH}$ \\
\hline$\hat{L}$ & $0.695 \mathrm{mH}$ & $0.705 \mathrm{mH}$ & \\
\hline
\end{tabular}

TABLE III

RESULTS UNDER ACCELERATION

\begin{tabular}{|c|c|c|c|}
\hline \multicolumn{4}{|c|}{ Sinusoidal EMF } \\
\hline \multicolumn{4}{|c|}{$\omega_{m}^{\prime}=3000 \mathrm{rads}^{-2}$} \\
\hline$\omega$ & $140 \mathrm{rads}^{-1}$ & 175 rads $^{-1}$ & $205 \mathrm{rads}^{-1}$ \\
\hline$\hat{R}$ & $0.0101 \Omega$ & $0.0102 \Omega$ & $0.0105 \Omega$ \\
\hline$\hat{L}$ & $0.709 \mathrm{mH}$ & $0.708 \mathrm{mH}$ & $0.709 \mathrm{mH}$ \\
\hline \multicolumn{4}{|c|}{ Modular EMF } \\
\hline \multicolumn{4}{|c|}{$\omega_{m}^{\prime}=3000 \mathrm{rads}^{-2}$} \\
\hline$\hat{R}$ & $0.0110 \Omega$ & $0.0115 \Omega$ & $0.0121 \Omega$ \\
\hline$\hat{L}$ & $0.695 \mathrm{mH}$ & $0.705 \mathrm{mH}$ & $0.725 \mathrm{mH}$ \\
\hline
\end{tabular}

case as the induced harmonics cannot be accounted for in the linearisation method. The problem becomes worse with speed increase,as shown in Table IV as the amplitude of the harmonics becomes greater whilst the resistive signal voltage remains constant.

Under acceleration there is a speed change during injection, which in the case of non-sinusoidal EMF increases the error formed when attempting to linearise for the motor normal operation described in Section VIII-B.

\section{Conclusion}

A resistance and inductance estimating method has been presented which has the potential to be applied on-line and with a minimum interruption to motor control. The zero-torque producing method of injection overcomes the limitations of direct-current injection methods, without compromising estimation accuracy and without the capacitor bank that would be required for a comparable zero-sequence based technique. The method has been developed to reduce power consumption and requires no more hardware than already necessary for a vector controlled drive system.

Power dissipation is shown to be minimal when the method is applied intermittently, sufficient for machines with long thermal time constants.

TABLE IV

NOISE PERFORMANCE

\begin{tabular}{|l|l|l|l|l|l|}
\hline$\sigma_{n}$ & $2 \times 10^{-5}$ & $2 \times 10^{-4}$ & $2 \times 10^{-3}$ & $2 \times 10^{-2}$ & $2 \times 10^{-1}$ \\
\hline$\hat{R}$ & $0.0108 \Omega$ & $0.0109 \Omega$ & $0.0110 \Omega$ & $0.0118 \Omega$ & $0.0140 \Omega$ \\
\hline
\end{tabular}

Machine EMF is shown to have a significant effect on resistance estimation accuracy, and limits application of the method to machines with EMF waveforms which are a good approximation to a pure sine wave.

Preliminary results from simulation have demonstrated the potential of the method and identified areas for future work, e.g. noise performance, and methods better able to separate the motor and injection voltages in the presence of non-sinusoidal EMF waveforms. A realisable practical system has further issues to consider, such as measurement of voltage from pulsewidth modulated phases, and instrumentation effects such as current transducer linearity and offset errors.

\section{REFERENCES}

[1] S. Farag, R. Bartheld, and T. Habetler, "An integrated on-line motor protection system," vol. 2(2), pp. 21-6, Mar/Apr. 1996.

[2] R. Kerkman, B. Seibel, T. Rowan, and D. Schlegel, "A new flux and stator resistance identifier for ac drive systems," IEEE Trans. Ind. Applicat., vol. 32, no. 3, pp. 585-593, May/June 1996.

[3] M. Elbuluk, L. Tong, and I. Hussain, "Neural-network-based model reference adaptive systems for high performance motor drives and motion controls," IEEE Trans. Ind. Applicat., vol. 38, no. 3, pp. 879886, June 2002.

[4] J. Campbell and M. Sumner, "An artificial neural network for stator resistance estimation in a sensorless vector controlled induction motor drive," in 9th European Conference on Power Electronics and Applications, Graz, Austria, Aug. 2001.

[5] L. Umanand and S. Bhat, "Online estimation of stator resistance of an induction motor for speed control applications," IEE Proc.-Electr: Power Appl., vol. 142, no. 2, pp. 97-103, Mar. 1995.

[6] V. Vasic, S. Vukosavic, and E. Levi, "A stator resistance estimation scheme for speed sensorless rotor flux oriented induction motor drive," IEEE Trans. Energy Conversion, vol. 18, no. 4, pp. 476-482, Dec. 2003.

[7] B. Bose and N. Patel, "Quasi-fuzzy estimation of stator resistance of induction motor," IEEE Trans. Power Electron., vol. 18, no. 4, pp. 401409, May 1998.

[8] S. Mir, M. Elbuluk, and D. Zinger, "Pi and fuzzy estimators for tuning the stator resistance in direct torque control of induction machines," IEEE Trans. Power Electron., vol. 13, no. 2, pp. 279-287, Mar. 1998.

[9] L. C. Benn, B. Burton, and R. G. Harley, "Online stator resistance estimation for thermal monitoring of converter fed induction motors using pseudorandom modified pwm," in Conference Record of the 2004 IEEE Industry Applications Conference. 39th LAS Annual Meeting, Oct. 2004, pp. 727-34.

[10] B. Nahid-Mobarakeh, F. Meibody-Tabar, and F.-M. Sargos, "Mechanical sensorless control of pmsm with online estimation of stator resistance," IEEE Trans. Ind. Applicat., vol. 40(2), pp. 457-71, Mar./Apr. 2004

[11] P. Milanfar and J. H. Lang, "Monitoring the thermal condition of permanent-magnet synchronous motors," IEEE Trans. Aerosp. Electron. Syst., vol. 32(4), pp. 1421-9, Oct. 1996.

[12] S. Lee and T. Habetler, "An online stator winding resistance estimation technique for temperature monitoring of line connected induction machines," IEEE Trans. Ind. Applicat., vol. 39, no. 3, pp. 685-694, June 2003.

[13] C. Jacobina, J. Filha, and A. Lima, "On-line estimation of the stator resistance of induction machines based on zero sequence model," IEEE Trans. Power Electron., vol. 15, no. 2, pp. 346-352, Mar. 2000.

[14] P. Stewart and V. Kadirkamanathan, "Dynamic model tracking design for low inertia, high speed permanent magnet ac motors," ISA Transactions, vol. 43, no. 1, pp. 111-22, Jan. 2004.

[15] S. D. Stearns and D. R. Hush, "Digital signal analysis," vol. 1990, p. 157, Prentice-Hall.

[16] A. V. Oppenheim and R. W. Schafer, Discrete-time signal processing. Prentice-Hall, 1998. 